\title{
Photoelectrochemical behavior of a rhodamine dye intercalated in a photocatalytically active layered niobate and photochemically inert clay
}

\author{
Ryota SHINOZAKI and Teruyuki NAKATO ${ }^{\dagger}$ \\ Division of Bio-Applications and Systems Engineering (BASE), Institute of Symbiotic Science and Technology, \\ Tokyo University of Agriculture and Technology, 2-24-16, Naka-cho, Koganei-shi 184-8588
}

\begin{abstract}
We investigated photoelectrochemical behavior of rhodamine 6G (R6G) dye intercalated in layered oxides of semiconducting hexaniobate and photochemically inert saponite clay. The R6G-clay intercalation compound generated comparable cathodic and anodic photocurrents. Photocurrent action spectra indicated that both the photocurrents were ascribed to photoexcitation of R6G monomers and dimers present in the interlayer spaces. On the other hand, the R6G-niobate intercalation compound exhibited a relatively large cathodic and small anodic photocurrents. Action spectra of the R6G-niobate system indicated that the photocurrents were generated by photoexcitation of only the R6G monomers while that the coexisting dimeric species were apparently silent. This result was explained by that the photocurrents were generated through photosensitization of the photocatalytically active semiconducting niobate layers with electron transfer from the photoexcited R6G monomers, whereas that the dimers were deactivated through dye-dye interactions.
\end{abstract}

(C2008 The Ceramic Society of Japan. All rights reserved.

Key-words : Layered niobate, Clay, Rhodamine 6G, Intercalation, Photoelectrochemical behavior, Sensitization

[Received November 20, 2007; Accepted January 17, 2008]

\section{Introduction}

Inorganic layered crystals provide two-dimensionally confined interlayer spaces. Guest molecules intercalated into the interlayer spaces of layered host crystals can exhibit unique properties because the guest species interact with both of the host layers sandwiching them and other intercalated molecules arranged in the interlayer spaces. ${ }^{1)}$ In particular, unusual photochemical/photophysical properties have been obtained for intercalation compounds of photofunctional molecules whose behavior is greatly affected by their microenvironments. Examples are peculiar spectroscopic properties, ${ }^{2)}$ photochromic behavior, ${ }^{3)-5)}$ regioselective photochemical reactions, ${ }^{6}$ photoinduced electron/energy transfer. ${ }^{7), 8)}$

Among inorganic layered crystals, layered niobates and titanates ${ }^{9)}$ are known as wide band-gap semiconductors possessing photocatalytic activities. ${ }^{10), 11)}$ Intercalation compounds of these oxides can provide electronic/energetic interactions between the semiconducting host layers and the guest molecules to exhibit unusual photochemical behaviors. Lead by such motivation, peculiar photochemical properties have been developed for intercalation compounds of layered niobates and titanates: e.g., photoinduced electron transfer and photosensitization. ${ }^{12)-19)}$ In particular, photosensitization of the semiconducting niobate and titanate layers has been recognized as an important challenge because these oxides become able to respond to visible light with the aid of photosensitizing dye species. ${ }^{14)-19)}$ In fact, the layered niobates and titanates intercalated with a ruthenium complex ${ }^{14), 15), 17), 18)}$ and porphyrin ${ }^{19)}$ have been utilized as photoelectrodes by which photoenergy of visible light is converted to electric current. However, variety of the dyes examined as photosensitizers of layered niobates and titanates has been rather

Corresponding author: T. Nakato; E-mail: tnakat@cc.tuat.ac.jp limited compared with sensitization of $\mathrm{TiO}_{2}$ that has vastly been investigated as a typical wide band-gap semiconductor possessing nonporous three dimensional structure. ${ }^{20)-22)}$

Among alternative photosensitizing dyes for the layered niobates and titanates, rhodamine dyes are attractive species because they have been reported to work as sensitizers of $\mathrm{TiO}_{2}{ }^{20), 23), 24)} \mathrm{In}$ addition, rhodamine dyes show peculiar photochemical/photophysical behaviors such as environment-sensitive spectroscopic properties, ${ }^{25)-27)}$ characteristic photodecomposition, ${ }^{28)-30)}$ and lasing capability. ${ }^{31)}$ Thus, it is worth investigating photochemical/ photophysical properties of the rhodamine dyes intercalated in the layered niobates and titanates. In fact, $\mathrm{we}^{32)}$ have investigated photodecomposition of cationic rhodamine $6 \mathrm{G}(\mathrm{R} 6 \mathrm{G})^{33)}$ incorporated into layered potassium hexaniobate $\mathrm{K}_{4} \mathrm{Nb}_{6} \mathrm{O}_{17}{ }^{34)}$ in comparison with that intercalated into photochemically inert saponite clay (a clay mineral of the smectite group). This study has indicated that the R6G species apparently exhibits similar photochemical behavior in both the layered hosts; the semiconducting host seems to contribute only slightly to the photoprocess of the intercalated dye molecules. However, we may observe photochemical communications in the R6G-niobate intercalation compound under appropriate conditions, because photoinduced electronic/energetic interactions should be allowed between the niobate layers and the R6G molecules if we consider previous studies of niobate-dye systems. ${ }^{17), 18)}$ Since the R6G molecules exhibit peculiar aggregative behavior in the interlayer spaces of niobate, ${ }^{35}$ the photochemical host-guest interactions may be related to the aggregated state of the intercalated dye species.

In the present study, we investigated photochemical process of the R6G dye intercalated in layered hexaniobate by using a photoelectrochemical technique, which can distinguish reactions in the interlayer spaces based on redox potential and current. Photocurrent action spectra showed that only monomeric R6G mol- 
ecules were active to sensitize the niobate layers although coexisting dimeric species were silent. Such behavior of the intercalated R6G species was different from that in photochemically inert saponite clay, demonstrating the presence of peculiar interactions between the semiconducting niobate layers and the rhodamine dye.

\section{Experimental}

2.1 Preparation of the intercalation compounds

The intercalation compounds of hexaniobate and saponite clay with R6G were prepared according to the methods reported previously. ${ }^{32), 35)}$ The R6G-niobate intercalation compound was synthesized with stepwise intercalation of propylammonium $\left(\mathrm{C}_{3} \mathrm{~N}\right)$ and $\mathrm{R} 6 \mathrm{G}$ cations into potassium hexaniobate $\mathrm{K}_{4} \mathrm{Nb}_{6} \mathrm{O}_{17} .^{35)}$ $\mathrm{K}_{4} \mathrm{Nb}_{6} \mathrm{O}_{17}$ prepared by a solid-state reaction ${ }^{36}$ was allowed to react with an excess amount of a $1 \mathrm{~mol} \mathrm{dm}^{-3}$ aqueous $\mathrm{C}_{3} \mathrm{~N}$ solution at room temperature for $90 \mathrm{~min}$. Then, the $\mathrm{C}_{3} \mathrm{~N}$-intercalated niobate was allowed to react with an excess amount of a $0.03 \mathrm{~mol}$ $\mathrm{dm}^{-3}$ aqueous R6G solution at $353 \mathrm{~K}$ for 3 weeks. The R6G-clay intercalation compound was prepared by a direct reaction of a synthetic saponite clay (Sumecton SA, Kunimine Industries; cat-

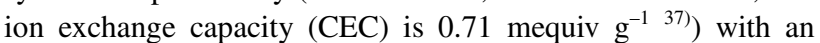
excess amount of a $0.01 \mathrm{~mol} \mathrm{dm}^{-3}$ aqueous R6G solution at room temperature for $1 \mathrm{~d}^{32)}$ Both the products were thoroughly washed with water and methanol.

Formation of the intercalation compounds was confirmed with powder X-ray diffraction (XRD), visible spectroscopy, and elemental analyses. XRD patterns were obtained with a MAC Science MX Labo diffractometer (monochromatic $\mathrm{CuK} \alpha$ radiation). Visible diffuse reflectance spectra were recorded on a Shimadzu UV-2450 spectrophotometer equipped with an integrating sphere. Prior to the spectral measurements, the samples were diluted with $\alpha-\mathrm{Al}_{2} \mathrm{O}_{3}$ powders (sample $/ \mathrm{Al}_{2} \mathrm{O}_{3}$ ratio was about 1/ 1000 in weight) to avoid saturation of the spectrum, and damped with a few water droplets to obtain the spectra under the conditions similar to those of photoelectrochemical measurements in aqueous solutions.

\subsection{Photoelectrochemical measurement}

Electrochemical measurements were performed with a conventional three-electrode cell in a $0.1 \mathrm{~mol} \mathrm{dm}^{-3} \mathrm{~K}_{2} \mathrm{SO}_{4}$ aqueous solution. A glass plate coated with indium tin oxide (ITO, $1 \times$ $1.5 \mathrm{~cm}^{2}$, EHC Co.) was used as the working electrode. Electrode potentials were measured with respect to an $\mathrm{Ag} / \mathrm{AgCl}\left(3 \mathrm{~mol} \mathrm{dm}^{-3}\right.$ $\mathrm{NaCl}$ aq.) reference electrode. A Pt black wire electrode was used as a counter electrode. Cyclic voltammograms were recorded by using a BAS ALS-401 electroanalyzer with a sweep rate of $10 \mathrm{mV} \mathrm{s}^{-1}$. To remove dissolved oxygen in the electrolyte solution, $\mathrm{N}_{2}$ gas was purged for 30 min before each measurement that was conducted under a flow of nitrogen.

The ITO working electrode was modified with the R6Gniobate or R6G-clay intercalation compound through the following procedure. Powders of the intercalation compound were suspended in toluene (for niobate) or water (for clay), and the suspension was drop-cast onto the electrode. Then, the electrode surface was coated with poly(methyl methacrylate) by drop-casting a toluene solution $(0.5$ mass $\%)$ in order to suppress leakage of the modified material during the photoelectrochemical measurements.

The modified ITO electrodes were subjected to choppered irradiation of visible light by using a $500 \mathrm{~W}$ Xe lamp (Ushio UXL-500SX) through a cutoff (> $460 \mathrm{~nm}$, Kenko Y-46) and heat-absorbing (Kenko HA-50) filters. The light was collected onto the electrode surface through a convex lens. Photocurrent action spectra were recorded at $-0.2 \mathrm{~V}$ or $0.6 \mathrm{~V}$ vs. $\mathrm{Ag} / \mathrm{AgCl}$ through irradiating the electrode with a $150 \mathrm{~W}$ Xe lamp (Ushio UXL-159) after passing a monochromater.

\section{Results and discussion}

\subsection{Characterization of the intercalation compounds}

Both the R6G-niobate and R6G-clay intercalation compounds prepared in the present study were identical to those reported in our previous papers. ${ }^{32), 35)}$ Figure 1 shows XRD patterns of the samples. The R6G-niobate intercalation compound gives the basal spacing of $3.22 \mathrm{~nm}$, corresponding to the gallery height of $1.58 \mathrm{~nm}$ (Fig. 1b). Elemental analyses have shown that the sample contains 0.41 R6G molecules in a unit cell of the niobate. These data clarify that a R6G molecule occupies $1.0 \mathrm{~nm}^{3}$ of the interlayer space $\left(1.7 \mathrm{~mol} \mathrm{dm}^{-3}\right)$. On the other hand, the R6G-clay intercalation compound gives the basal spacing of $1.87 \mathrm{~nm}$, indicating the gallery height of $0.91 \mathrm{~nm}$ (Fig. 1d). The amount of R6G dye intercalated has been estimated to be $0.83 \mathrm{mmol}$ per one gram of clay whose theoretical surface area is $750 \mathrm{~m}^{2} \mathrm{~g}^{-1}{ }^{8)}$

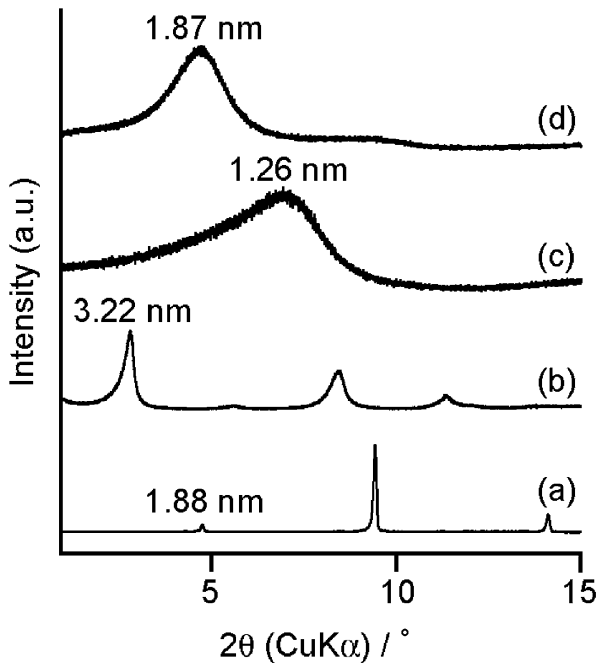

Fig. 1. XRD patterns of (a) $\mathrm{K}_{4} \mathrm{Nb}_{6} \mathrm{O}_{17} \cdot 3 \mathrm{H}_{2} \mathrm{O}$, (b) the R6G-niobate intercalation compound, (c) saponite clay, and (d) the R6G-clay intercalation compound.

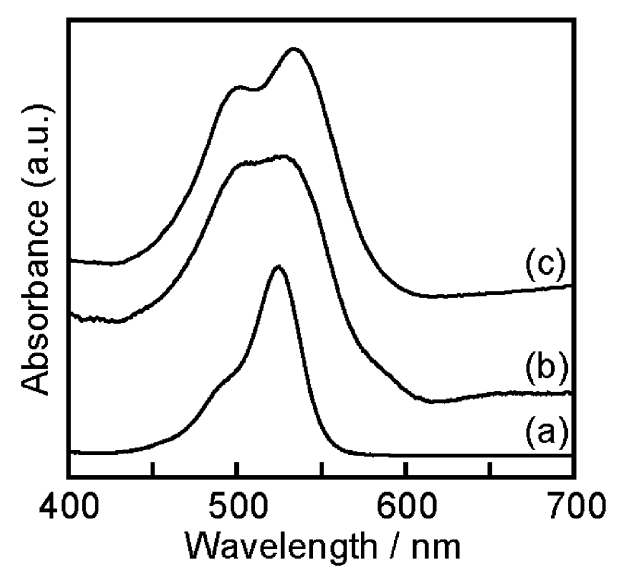

Fig. 2. (a) Absorption spectrum of (a) an aqueous R6G solution $\left(10^{-6}\right.$ $\mathrm{mol} \mathrm{dm}{ }^{-3}$ ), and diffuse reflectance spectra of (b) the R6G-niobate intercalation compound and (c) the R6G-clay intercalation compound. 
These values give the interlayer space occupied by a R6G mol-

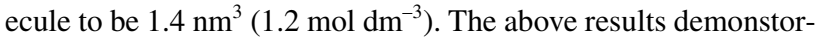
ate that the dye molecules are densely accommodated in the interlayer spaces of both hexaniobate and saponite clay.

The dense accommodation of R6G in the interlayer spaces of niobate and clay was reflected by aggregation of the dye molecules as characterized spectroscopically. Visible diffuse reflectance spectrum of the R6G-niobate intercalation compound shown in Fig. 2b indicates the coexistence of R6G monomers and H-dimers ${ }^{25-27), 33), 38)}$ evidenced by the absorption maxima at $530 \mathrm{~nm}$ and $507 \mathrm{~nm}$, respectively, with comparable amounts by considering their extinction coefficients in aqueous solutions. ${ }^{33)}$ Spectrum of the R6G-clay intercalation compound also exhibits absorption bands of R6G monomers and $\mathrm{H}$-dimers at $538 \mathrm{~nm}$ and $505 \mathrm{~nm}$, respectively, as shown in Fig. 2c. The spectroscopic results demonstrate that the intercalated $\mathrm{R} 6 \mathrm{G}$ molecules were aggregated to large extents to form $\mathrm{H}$-dimers in the interlayer spaces of niobate and clay.

\subsection{Photoelectrochemical behavior of the R6G-clay intercalation compound}

The R6G dye intercalated in saponite clay exhibited photoelectrochemical response upon irradiation of visible light. Figure 3 shows a cyclic voltammogram recorded under choppered irradiation of the ITO electrode modified with the R6G-clay intercalation compound. Both cathodic and anodic photocurrents are observed at the potential regions of more negative than $-0.1 \mathrm{~V}$ vs $\mathrm{Ag} / \mathrm{AgCl}$ and more positive than $0 \mathrm{~V}$ vs $\mathrm{Ag} / \mathrm{AgCl}$, respectively. Since the saponite clay is photochemically inert, the photoelectrochemical response must be induced by photoexcitation of the intercalated R6G species. This is confirmed by the photocurrent action spectra shown in Fig. 4. Both the cathodic and anodic photocurrents show the same action spectra, which are consistent with the absorption spectrum of the intercalation compound. A peak at $530 \mathrm{~nm}$ and a shoulder at around $500 \mathrm{~nm}$ correspond to the absorption bands of monomer and H-dimer, respectively, of the R6G dye. ${ }^{25)-27), 33), 38)}$ Hence, both the photocurrents observed for the R6G-clay intercalation compound are generated with the same origin, i.e., photoexcitation of the monomeric and dimeric R6G species immobilized in the interlayer space.

These results suggest that the R6G cations are transformed to

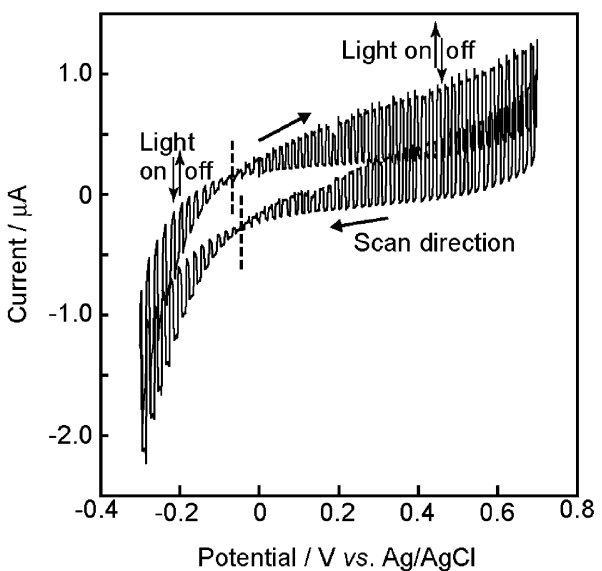

Fig. 3. Cyclic voltammogram of the R6G-clay intercalation compound loaded on an ITO electrode under choppered irradiation of visible light. The dashed lines indicate the potentials where the photocurrent changes from cathodic to anodic.
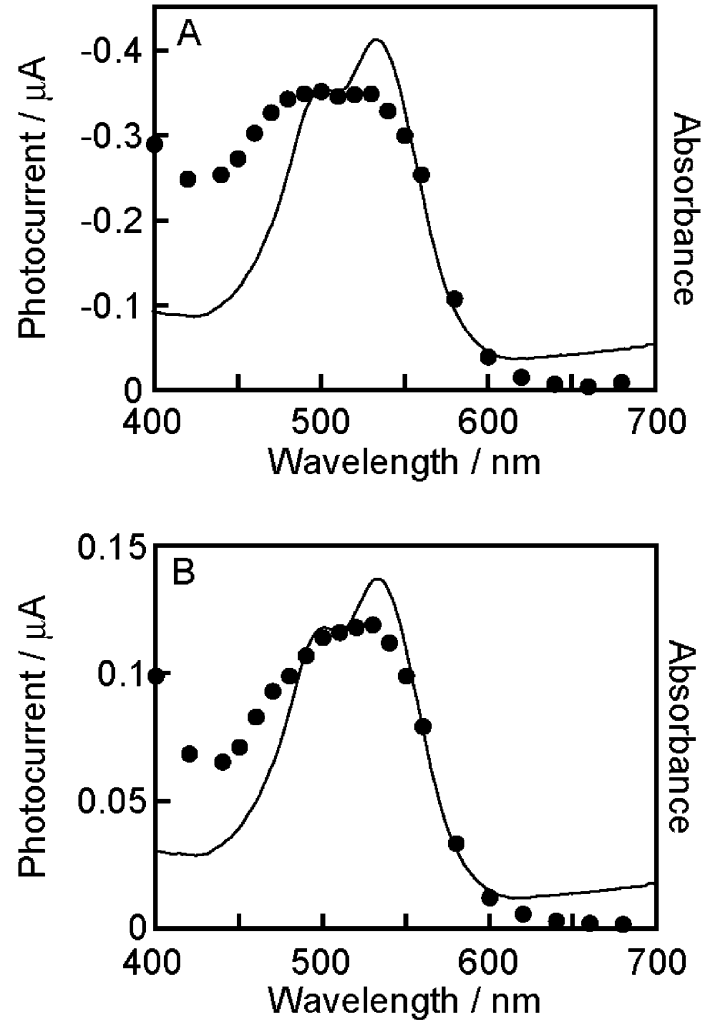

Fig. 4. Photocurrent action spectra of the R6G-clay intercalation compound at (A) -0.2 and (B) $0.6 \mathrm{~V}$ vs. $\mathrm{Ag} / \mathrm{AgCl}$. The action spectra indicated by the circles are given with the diffuse reflectance spectrum of the intercalation compound shown by the full line.

both reducible and oxidizable species through photoexcitation within saponite clay. We suppose photochemical disproportionation of R6G reported by Mialocq et al. ${ }^{38)}$ as the origin of the photocurrents. This process is described as a reaction of photoexcited R6G molecule (R6G ${ }^{*}$ ) with another ground state molecule yielding a pair of cationic and anionic R6G radicals, which will be written as $\mathrm{R}_{6} \mathrm{G}^{+\bullet}$ and $\mathrm{R}_{6} \mathrm{G}^{-\bullet}$ hereafter, as shown by eqn. $1.8)$

$$
\mathrm{R} 6 \mathrm{G}^{*}+\mathrm{R} 6 \mathrm{G} \rightarrow \mathrm{R} 6 \mathrm{G}^{+\bullet}+\mathrm{R}^{-\mathrm{G}^{-}}
$$

Then, the photochemically produced radical species are reduced and oxidized at the electrode to give the cathodic and anodic photocurrents, respectively, as indicated by eqn. 2 and 3 .

$$
\begin{aligned}
& {\mathrm{R} 6 \mathrm{G}^{+\bullet}}^{+\mathrm{e}^{-}} \rightarrow \mathrm{R} 6 \mathrm{G} \text { (cathodic photocurrent) } \\
& {\mathrm{R} 6 \mathrm{G}^{-}}^{-} \rightarrow \mathrm{R} 6 \mathrm{G}+\mathrm{e}^{-} \text {(anodic photocurrent) }
\end{aligned}
$$

Figure 5 illustrates a possible scheme of the photocurrent generation. Under the cathodic polarization $(<-0.1 \mathrm{~V}$ vs $\mathrm{Ag} / \mathrm{AgCl})$, the reduction of $\mathrm{R}_{6 \mathrm{G}}{ }^{+}$species (eqn. 2) preferentially occurs to give the cathodic photocurrent (Fig. 5a). When the electrode potential becomes positive ( $>0 \mathrm{~V}$ vs $\mathrm{Ag} / \mathrm{AgCl}$ ), the oxidation of $\mathrm{R} 6 \mathrm{G}^{-}$species (Eq. (3)) proceeds superiorly to generate the anodic photocurrent (Fig. 5b). This reaction sequence requires dense presence of R6G molecules because a photoexcited R6G molecule must react with another dye molecule located in proximity at the initial step (Eq. (1)). This requisite is satisfied in the R6G-clay intercalation compound since the interlayer space densely accommodates the dye molecules to dimerize them as mentioned above. 
(a) Cathodic photocurrent

(b) Anodic photocurrent
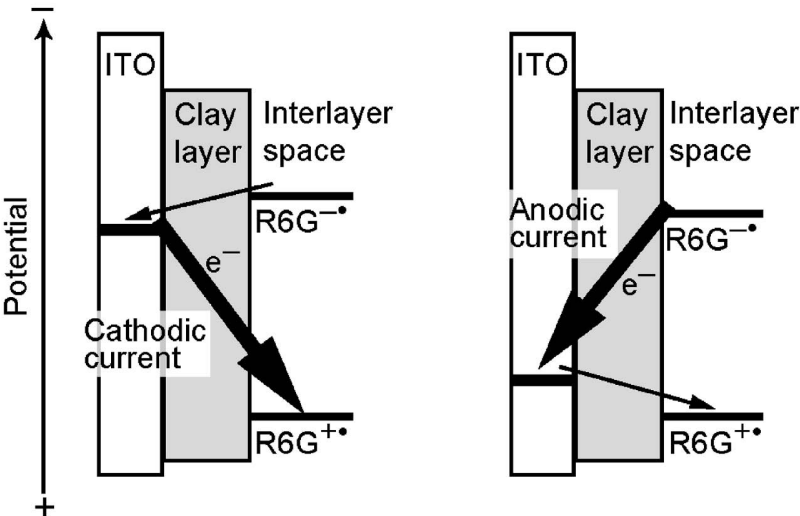

Fig. 5. Schematic energy diagram and a possible mechanism for the generation of (a) cathodic and (b) anodic photocurrents by the R6G-clay intercalation compound.

\subsection{Photoelectrochemical behavior of the R6G-niobate intercalation compound}

Photoelectrochemical response of the R6G-niobate intercalation compound was different from that of the dye within the clay. Figure 6 shows a cyclic voltammogram of the intercalation compound placed on an ITO electrode recorded under choppered irradiation of visible light. Although the R6G molecules can be slowly photodecomposed (N-de-ethylated) in the interlayer space of hexaniobate under anaerobic conditions as reported by us previously, ${ }^{32)}$ we confirmed by using visible spectroscopy that the photodecomposion of dye was negligible during the photoelectrochemical measurements in the present study. The voltammogram indicates a relatively large cathodic and a small anodic photocurrents whereas the R6G-clay intercalation compound gives comparable anodic and cathodic photocurrents. The photocurrents observed for the R6G-niobate intercalation compound were much smaller than those of the R6G-clay system although both the intercalation compounds accommodate the dye molecules in their interlayer spaces with similar densities. This would be ascribed to photoelectrochemical inactivity of the R6G dimers attached to the semiconducting niobate layers as discussed below and technical reasons such as difference in light scattering

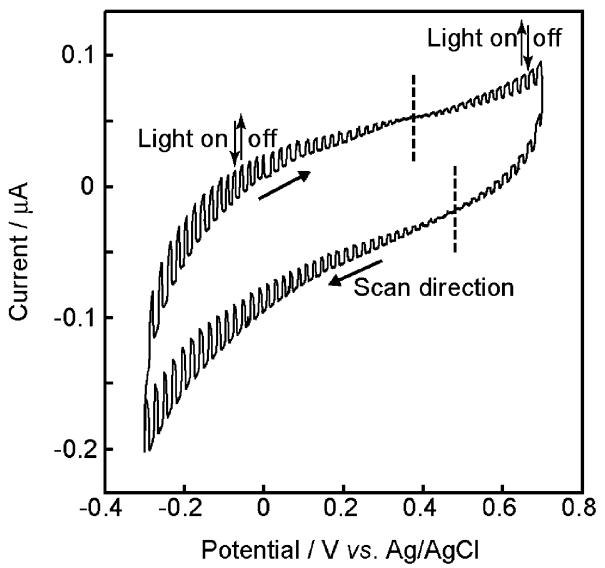

Fig. 6. Cyclic voltammogram of the R6G-niobate intercalation compound loaded on an ITO electrode under choppered irradiation of visible light. The dashed lines indicate the potentials where the photocurrent changes from cathodic to anodic.
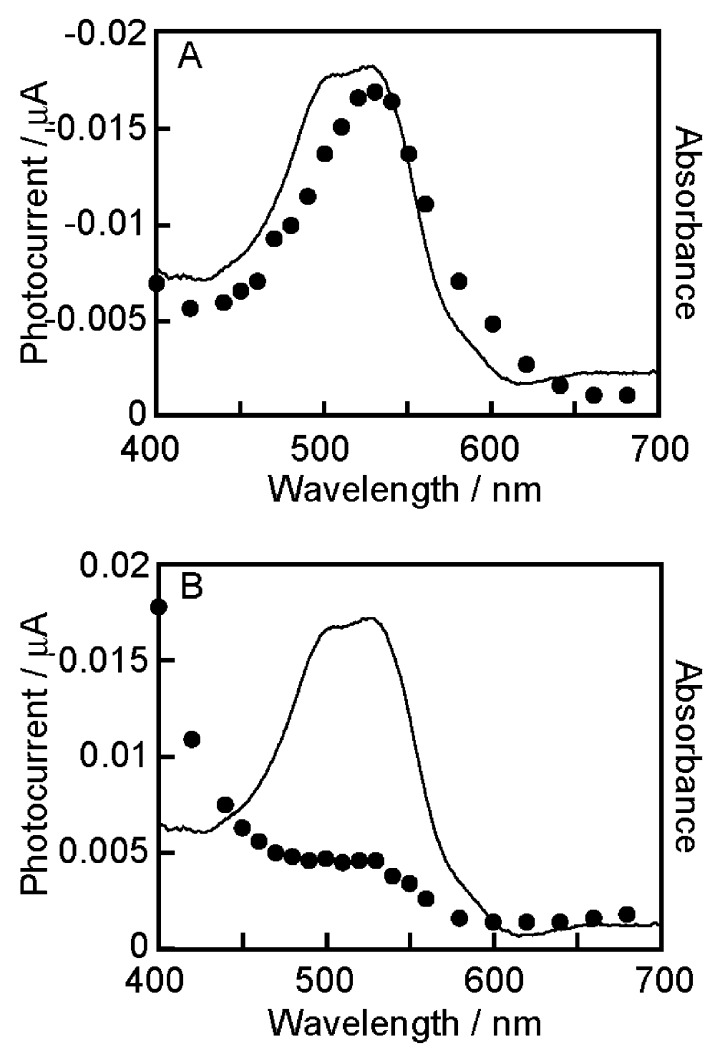

Fig. 7. Photocurrent action spectra of the R6G-niobate intercalation compound at (A) -0.2 and (B) $0.6 \mathrm{~V}$ vs. $\mathrm{Ag} / \mathrm{AgCl}$. The action spectra indicated by the circles are given with the diffuse reflectance spectrum of the intercalation compound shown by the full line.

Photocurrent action spectra of the R6G-niobate intercalation compound demonstrated that the photoresponse was caused by photoexcitation of the intercalated R6G dye. Figure 7A shows the action spectrum of the cathodic photocurrent. The spectrum gives a peak at $530 \mathrm{~nm}$ corresponding to the absorption of monomeric R6G species. ${ }^{25)-27), 33), 38)}$ However, it does not exhibit a peak or shoulder corresponding to the dimeric species, which is different from the photocurrent action spectra of the R6G-clay intercalation compound. The action spectrum of the anodic photocurrent shown in Fig. 7B also exhibits a broad band that can be ascribed to the monomer absorption band of R6G, although the current is much weaker than the cathodic photocurrent.

These results demonstrate that the photocurrents of the R6Gniobate intercalation compound are generated by photoexcitation of the R6G molecules present as monomers in the interlayer space while that the dimers coexisting with the monomers are photochemically silent. The absence of the photoresponse of dimers is not explained by the intrinsic nature of the R6G species because the photocurrents due to the photoexcitation of dimers are observed in the clay system; thus, the niobate layers contribute to this behavior. We assume that the R6G radical pair produced with photoexcitation of the rhodamine dimers (Eq. (4) $)^{38)}$ is deactivated through charge recombination (Eq. (5) $)^{38)}$ before transferring electrons to/from the electrode.

$$
\begin{aligned}
& (\mathrm{R} 6 \mathrm{G})_{2} \stackrel{h v}{\longrightarrow}\left(\mathrm{R} 6 \mathrm{G}^{*}+\mathrm{R} 6 \mathrm{G}\right) \rightarrow\left(\mathrm{R} \mathrm{G}^{+\bullet}+\mathrm{R}^{-\mathrm{G}^{-\bullet}}\right) \\
& \left(\mathrm{R} 6 \mathrm{G}^{+\bullet}+\mathrm{R} 6 \mathrm{G}^{-\bullet}\right) \rightarrow(\mathrm{R} 6 \mathrm{G})_{2}
\end{aligned}
$$

The niobate layers should allow electron migration because of 
their semiconducting property ${ }^{39)}$ to facilitate the charge recombination of the rhodamine radical pair. In contrast, the R6G radical species formed in the interlayer space of clay undergo electron transfer with the electrode to generate the photocurrents since the charge recombination passing along the oxide layers should be suppressed by the insulating clay layers.

We suppose that the cathodic photocurrent due to the monomeric R6G species is generated by photosensitization of the semiconducting niobate layers with the intercalated dye molecules accompanied by electron transfer between them. Since the R6G molecules are densely accommodated in the interlayer space of niobate, the photosensitizing interactions between the niobate layers and intercalated R6G molecules will compete with the interactions among the R6G molecules. The R6G dimers should be less advantageous than the monomers for the interactions with the niobate layers; the dimeric species should be favorable for the formation of R6G radical pair through the dye-dye interactions. Such consideration explains the little contribution of the R6G dimers to the cathodic photocurrents observed in the R6G-niobate system.

Although photosensitization of bulk semiconductors such as $\mathrm{TiO}_{2}$ with organic dyes usually generates anodic photocurrent, cathodic currents by photosensitization has been reported for the layered semiconducting oxides (hexaniobate and lepidocrocitetype titanate) with a ruthenium complex or methylene blue that is immobilized in their interlayer spaces. ${ }^{14), 18)}$ We assume a similar mechanism for the R6G-niobate system, and Fig. 8 illustrates the scheme of photocurrent generation. In this scheme, photoexcited R6G species (R6G ${ }^{*}$ ) reacts with the niobate layers to reduce them, by which the dye is oxidized to the radical cation R6G ${ }^{+\bullet}$ (Eq. (6)).

$$
\begin{aligned}
& \mathrm{R} 6 \mathrm{G}^{*}+\text { niobate } \rightarrow \mathrm{R}^{\circ} \mathrm{G}^{+\bullet}+\text { niobate }\left(\mathrm{e}^{-}\right) \\
& \left(\text {niobate }\left(\mathrm{e}^{-}\right) \text {: reduced niobate layers }\right)
\end{aligned}
$$

This is rationalized by that the redox potential of $\mathrm{R} 6 \mathrm{G}^{*}$, reported to be $-1.78 \mathrm{~V}$ vs $\mathrm{Ag} / \mathrm{AgCl},{ }^{40)}$ allows electron injection into the conduction band of hexaniobate $(-0.96 \mathrm{~V}$ vs $\mathrm{Ag} / \mathrm{AgCl}) .{ }^{41)}$ Then, the $\mathrm{R} 6 \mathrm{G}^{+\bullet}$ species will generate the cathodic current as in the case of the R6G-clay intercalation compound (Eq. (2)). On the other hand, the electrons transferred from the $\mathrm{R} 6 \mathrm{G}^{*}$ species to the niobate layers (Eq. (6)) can be hopped to the electrode to generate an anodic current. We assume that the small anodic photocurrent in the voltammogram (Fig. 6) is ascribed to this process (a) Cathodic photocurrent

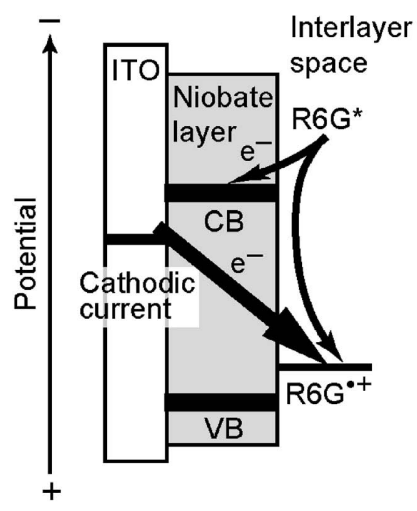

(b) Anodic photocurrent

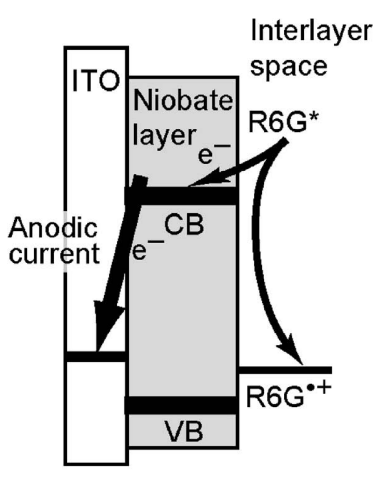

Fig. 8. Schematic energy diagram and a possible mechanism for the generation of (a) cathodic and (b) anodic photocurrents by the R6G-niobate intercalation compound.
(Eq. (7)).

$$
\text { niobate }\left(\mathrm{e}^{-}\right) \rightarrow \text { niobate }+\mathrm{e}^{-} \text {(anodic photocurrent) }
$$

However, this reaction competes with consumption of the electrons by protons in the electrolyte solution (Eq. (8)). ${ }^{14), 18)}$

$$
\text { niobate }\left(\mathrm{e}^{-}\right)+\mathrm{H}^{+} \rightarrow \text { niobate }+\frac{1}{2} \mathrm{H}_{2}
$$

Competition of the redox reactions of Eqs. 7 and 8 would rationalize the lowered anodic photocurrent shown in the voltammogram.

\section{Conclusions}

Photoelectrochemical measurements have clarified differences in the photochemical response of the intercalated R6G species depending on the host materials. The R6G species incorporated into the photochemically inert saponite clay exhibits photocurrents due to the photoresponse of the dye molecules themselves. The dense accommodation of the R6G molecules evidenced by the presence of dimeric species assists the dye-dye interactions responsible for the photocurrents. In contrast, the behavior of R6G species intercalated in the semiconducting niobate is explained by the photochemical interactions between the dye and the photocatalytically active oxide layers. In this system, the dimers are apparently photoelectrochemically inactive; thus, the dense confinement in the interlayer space is not helpful for the photochemical communication between the niobate layers and the dye molecules.

\section{References}

1) M. Ogawa and K. Kuroda, Chem. Rev., 95, 399-438 (1995).

2) M. Ogawa, R. Kawai and K. Kuroda, J. Phys. Chem., 100, 16218-16221 (1996).

3) T. Seki and K. Ichimura, J. Polym. Sci. Technol., 2, 147-152 (1989).

4) M. Ogawa, K. Fujii, K. Kuroda and C. Kato, Mater. Res. Soc. Symp. Proc., 233, 89-94 (1991).

5) R. Sasai, H. Itoh, I. Shindachi, T. Shichi and K. Takagi, Chem. Mater., 13, 2012-2016 (2001).

6) K. Takagi, H. Usami, H. Fukaya and Y. Sawaki, J. Chem. Soc., Chem. Commun., 1174-1175 (1989).

7) H. Miyata, Y. Sugahara, K. Kuroda and C. Kato, J. Chem. Soc., Faraday Trans. 1, 83, 1851-1858 (1987).

8) S. Takagi, D. A. Tryk and H. Inoue, J. Phys. Chem. B, 106, 5455-5460 (2002).

9) A. Clearfield, "Progress in Intercalation Research," Ed. by W. Müller-Warmuth and R. Schöllhorn, Kluwer, Dordrecht, (1994) pp. 223-271.

10) A. Kudo, J. Ceram. Soc. Japan, 109, S81-S88 (2001).

11) Y. Yamashita, K. Hyuga, V. Petrykin, M. Kakihana, M. Yoshimura, K. Domen, H. Kato and A. Kudo, J. Ceram. Soc. Japan, 115, 511-513 (2007).

12) H. Miyata, Y. Sugahara, K. Kuroda and C. Kato, J. Chem. Soc., Faraday Trans. 1, 84, 2677-2682 (1988).

13) T. Nakato, K. Kuroda and C. Kato, Chem. Mater., 4, 128-132 (1992).

14) U. Unal, Y. Matsumoto, N. Tanaka, Y. Kimura and N. Tamoto, J. Phys. Chem. B, 107, 12680-12689 (2003).

15) A. Ishikawa, T. Takata, J. N. Kondo, M. Hara, Y. Matsumoto and K. Domen, J. Mater. Res., 19, 661-666 (2004).

16) N. Miyamoto, K. Kuroda and M. Ogawa, J. Phys. Chem. B, 108, 4268-4274 (2004).

17) Z. Tong, S. Takagi, H. Tachibana, K. Takagi and H. Inoue, $J$. Phys. Chem. B, 109, 21612-21617 (2005).

18) U. Unal, Y. Matsumoto, N. Tamoto, M. Koinuma, M. Machida and K. Izawa, J. Solid State Chem., 179, 33-40 (2006).

19) K. Akatsuka, Y. Ebina, M. Muramatsu, T. Sato, H. Hester, D. 
Kumaresan, R. H. Schmehl, T. Sasaki and M. Haga, Langmuir, 23, 6730-6736 (2007).

20) T. Osa and M. Fujihira, Nature, 264, 349-350 (1976).

21) B. O'Regan and M. Grätzel, Nature, 353, 737-740 (1991).

22) P. V. Kamat, Chem. Rev., 93, 267-300 (1993).

23) T. Shimidzu, T. Iyoda and Y. Koide, J. Am. Chem. Soc., 107, 35-41 (1985).

24) R. Abe, K. Hara, K. Sayama, K. Domen and H. Arakawa, J. Photochem. Photobiol. A, 137, 63-69 (2000).

25) F. López Arbeloa, M. J. Tapia Estévez, T. López Arbeloa and I. López Arbeloa, Clay Miner., 32, 97-106 (1997).

26) F. del Monte, J. D. Mackenzie and D. Levy, Langmuir, 16, 7377-7382 (2000).

27) R. Sasai, T. Fujita, N. Iyi, H. Itoh and K. Takagi, Langmuir, 18, 6578-6583 (2002).

28) T. Watanabe, T. Takizawa and K. Honda, J. Phys. Chem., 81, 1845-1851 (1977).

29) J. Zhao, T. Wu, K. Wu, K. Oikawa, H. Hidaka and N. Serpone, Environ. Sci. Technol., 32, 2394-2400 (1998).

30) H. Park and W. Choi, J. Phys. Chem. B, 109, 11667-11674 (2005).
31) F. López Arbeloa, A. Costela and I. López Arbeloa, J. Photochem. Photobiol. A, 55, 97-103 (1990).

32) R. Shinozaki and T. Nakato, Micropor. Mesopor. Mater., in press (DOI:10.1016/j.micromeso.2007.1011.1005).

33) F. Lopez Arbeloa, I. Llona Gonzalez, P. Ruiz Ojeda and I. Lopez Arbeloa, J. Chem. Soc., Faraday Trans 2, 78, 989-994 (1982).

34) M. Gasperin, Acta Cryst. B, 38, 2024-2026 (1982).

35) R. Shinozaki and T. Nakato, Langmuir, 20, 7583-7588 (2004).

36) K. Nassau, J. W. Shiever and J. L. Bernstein, J. Electrochem. Soc., 116, 348-353 (1969).

37) N. Kakegawa, T. Kondo and M. Ogawa, Langmuir, 19, 3578$3582(2003)$.

38) J. C. Mialocq, P. Hébert, X. Armand, R. Bonneau and J. P. Morand, J. Photochem. Photobiol. A, 56, 323-338 (1991).

39) A. Furube, T. Shiozawa, A. Ishikawa, A. Wada, K. Domen and C. Hirose, J. Phys. Chem. B, 106, 3065-3072 (2002).

40) L. Bahadur and P. Srivastava, Sol. Energy Mater. Sol. Cells, 79, 235-248 (2003).

41) Y. I. Kim, S. J. Atherton, E. S. Brigham and T. E. Mallouk, J. Phys. Chem., 97, 11802-11810 (1993). 